\title{
SIMPLE GOING DOWN IN PI RINGS
}

\author{
PHILLIP LESTMANN 1
}

\begin{abstract}
In this paper we prove two generalizations of a theorem which McAdam proved for commutative rings. Theorem 1 states that if $R \subset S$ is a central integral extension of PI rings, then going down for prime ideals holds between $R$ and $S$ if and only if going down holds in $R \subset R[s]$ for each $s \in S$. Theorem 2 gives the analogous result for going down in $C \subset R$ where $C$ is a central subring of the PI ring $R$. As a corollary we obtain a result of Schelter generalizing Krull's theorem on going down for integral extensions of integrally-closed subrings.
\end{abstract}

If $R$ is a ring satisfying a polynomial identity, we shall say that $R$ is a PI ring. A pair of rings $R \subset S$ is said to have going down (GD) if for every pair of prime ideals $P_{1} \subset P$ of $R$ and every prime ideal $Q$ of $S$ with $Q \cap R=P$, there exists a prime ideal $Q_{1}$ of $S$ so that $Q_{1} \subset Q$ and $Q_{1} \cap R=P_{1}$. The pair $R \subset S$ is said to have simple going down (SGD) if $R \subset R[t]$ has GD for each $t \in S$.

If $R \subset S$ is a pair of rings, $S$ is said to be integral over $R$ if each element $S$ of $S$ satisfies an equation of the form $s^{n}+r_{1} s^{n-1}+\cdots+r_{n-1} s+r_{n}=0$ where $r_{i} \in R, 1 \leqslant i \leqslant n$. McAdam has shown [1] the following: If $R \subset T$ is a pair of commutative rings and $T$ is integral over $R$, then $R \subset T$ has GD if and only if $R \subset T$ has SGD. ${ }^{2}$ To extend this idea we first need another definition.

Definition. For any rings $R \subset S$ let $S^{R}=\{s \in S \mid s r=r s$ for each $r \in R\}$. We say that $R \subset S$ is an extension if $S=R S^{R}$. The extension is said to be central if $S=R Z(S)$ where $Z(S)=$ the center of $S$.

In this paper we generalize McAdam's result in Theorems 1 and 2 as follows:

THeOREM 1. Let $R \subset S$ be a central integral extension of PI rings. Then $R \subset S$ has GD if and only if $R \subset S$ has SGD.

Received by the editors August 6, 1976.

AMS (MOS) subject classifications (1970). Primary 16A38, 16A66.

Key words and phrases. PI ring, going down, simple going down, integral, extension, going up, lying over, incomparability.

1 This paper is part of the author's thesis for the Ph.D. degree at the University of Southern California.

2 The author is indebted to the referee for pointing out that this result has appeared as Proposition 2 in McAdam, Going down and open extensions, Canad. J. Math. 27 (1975), 111-114. 
TheOREM 2. Let $R$ be a PI ring integral over the central subring $C$. Then $C \subset R$ has GD if and only if $C \subset R$ has SGD.

As a corollary to Theorem 2 we get the generalization of Krull's going down theorem for integral extensions of integrally-closed subrings which was proved by Schelter [4].

We begin with the following definition by way of reminder.

Definition. Let $R \subset S$ be a pair of rings.

(1) If for each prime $P$ of $R$ there exists a prime $Q$ of $S$ so that $Q \cap R=P$, then $R \subset S$ is said to have lying over (LO).

(2) If for any pair of primes $P \subset P_{1}$ of $R$ and prime $Q$ of $S$ with $Q \cap R=P$ there is a prime $Q_{1}$ of $S$ with $Q \subset Q_{1}$ and $Q_{1} \cap R=P_{1}$, then $R \subset S$ is said to have going up $(\mathrm{GU})$.

(3) If $P$ any prime of $R$ and $Q_{1}$ and $Q_{2}$ primes of $S$ with $Q_{1} \cap R=Q_{2} \cap R$ $=P$ implies that $Q_{1} \nsubseteq Q_{2}$ and $Q_{2} \nsubseteq Q_{1}$, then $R \subset S$ is said to have incomparability (INC).

Lemma 1. Let $R$ be any ring and let $B$ be a commutative subring of $R$. Assume $R$ is integral over $B$. If $P$ is any prime ideal of $B$ and $z \in P$, then $z R \cap B \subseteq P$.

Proof. Take $z \in P$ and suppose $z r \in B$ for some $r \in R$. $R$ is integral over $B$; so we have an equation $r^{n}+b_{1} r^{n-1}+\cdots+b_{n}=0$ for some $b_{i} \in B, 1$ $\leqslant i \leqslant n$. Since $z r \in B$ and $B$ is commutative, $z r z=z^{2} r$. Hence, we may write

$$
0=z^{n}\left(r^{n}+b_{1} r^{n-1}+\cdots+b_{n}\right)=(z r)^{n}+b_{1} z(z r)^{n-1}+\cdots+b_{n} z^{n} .
$$

Thus $(z r)^{n} \in P$, and so $z r \in P$ since $P$ is a prime ideal of the commutative ring $B$.

The following lemma is Theorem 1 of $[3]:^{3}$

Lemma 2. If $R \subset S$ is an integral extension and $R$ satisfies a polynomial identity, then $R \subset S$ has $\mathrm{GU}, \mathrm{INC}$, and LO.

The next lemma is not new, but it is added here for completeness.

Lemma 3. If $R$ is any ring and $P$ is a minimal prime ideal of $R$, then $P \cap Z(R)$ consists of zero-divisors of $R$.

Proof. Let $A_{0}=\{r \in R \mid r$ is regular in $R$ (i.e., $r$ has no nontrivial right or left annihilator in $R)\}$, and set $A=A_{0} \cap Z(R)$. If $A_{0} \cap Z(R)=\varnothing$, we are done trivially. Let $B=R-P$. Write $A B=\{a b \mid a \in A, b \in B\}$. Note that $0 \notin A B$ since $0 \notin B$ and $A$ consists of regular elements. Let $Q$ be an ideal of $R$ maximal with respect to the property $Q \cap A B=\varnothing$. Claim. $Q$ is a prime

${ }^{3}$ Schelter has issued a correction to his paper which generalizes the definition of integral. He defines an element $s$ of an extension of a ring $R$ to be integral over $R$ if $s$ satisfies a monic polynomial in $R \stackrel{*}{\subset} C[x]$, the free product of $R$ and $C[x]$, where $C$ is the center of $R$. With a slight change in our proof of Lemma 1, the results of this paper hold for this more general (and useful) definition of integral. 
ideal of $R$. If not, there exist ideals $I, J$ of $R$ with $Q \varsubsetneqq I, Q \varsubsetneqq J$, and $I J \subseteq Q$. By maximality of $Q$ we may take $x \in I \cap A B$ and $y \in J \cap A B$. We have $x, y \in R-Q$ and $x R y \subseteq Q$. Since $x, y \in A B$, we may set $x=a_{1} b_{1}$ and $y=a_{2} b_{2}$, where $a_{1}, a_{2} \in A, b_{1}, b_{2} \in B$. Since $b_{1}, b_{2} \notin P$, there is an $r \in R$ so that $b_{1} r b_{2} \notin P$. But $x r y=a_{1} b_{1} r a_{2} b_{2}=\left(a_{1} a_{2}\right)\left(b_{1} r b_{2}\right) \in A B \cap Q$. Contradiction. So $Q$ is a prime ideal of $R$.

Suppose $Q \nsubseteq P$. Take $b \in Q-P$ and any $a \in A$. This gives $a b \in Q$ $\cap A B$. So we must have $Q \subseteq P$. This implies that $Q=P$ since $P$ is a minimal prime. Now suppose $Q \cap A \neq \varnothing$ and take $a \in Q \cap A$. If $b \in B$ we again have the contradiction $a b \in Q \cap A B$. Therefore $\varnothing=Q \cap A=P \cap A$, and we are done.

The next lemma was proved by McAdam for $R \subset S$ both commutative in [1].

Lemma 4. Let $R \subset S$ be any extension of $\mathrm{PI}$ rings, and let $P \subset P_{1}$ be prime ideals of $R$. Suppose $Q_{1}$ is a prime of $S$ with the property that $Q_{1} \cap R=P_{1}$. Set $W=\left\{Q_{\alpha} \mid Q_{\alpha}\right.$ is a prime ideal of $\left.S, Q_{\alpha} \cap R=P\right\}$, and define $I=\cap\left\{Q_{\alpha} \mid Q_{\alpha}\right.$ $\in W\}$. We assume $W \neq \varnothing$. Then there is a prime $Q$ of $S$ with $Q \subset Q_{1}$ and $Q \cap R=P$ if and only if $I \subset Q_{1}$.

Proof. $(\Rightarrow)$ Obvious.

$(\Leftarrow)$ Suppose $I \subset Q_{1}$. Then $P \subset I \subset Q_{1}$. Let $Q$ be a prime ideal of $S$ in $Q_{1}$ minimal over $P$. If $Q \cap R \neq P$, then $\bar{Q} \cap \bar{R} \neq \overline{0}$ in $\bar{S}=S / I$. Now $\bar{R} \subset \bar{S}$ is still an extension of PI rings. Since $I \cap R=P, \bar{R}$ is a prime PI ring. So there is a $\bar{q} \in Z(\bar{R}) \cap \bar{Q}$ with $\bar{q} \neq \overline{0}$ [5]. Since $Z(\bar{R}) \subset Z(\bar{S})$ and $\bar{Q} \cap Z(\bar{S})$ consists of zero-divisors by Lemma 3 , we can find a $\bar{t} \neq \overline{0}$ in $\bar{S}$ so that $\overline{q t}=\overline{0}$. However, $\bar{t} \neq \overline{0}$ gives a $\bar{Q}_{\alpha}$ for which $\bar{t} \notin \bar{Q}_{\alpha}$. This forces $\bar{q} \in \bar{Q}_{\alpha}$. If not, $\bar{S} / \bar{Q}_{\alpha}$ is a prime PI ring with a nonzero central element which is a zero-divisor. Thus $\bar{q}$ is in $\bar{Q}_{\alpha} \cap \bar{R}=\overline{0}$ since $Q_{\alpha} \cap R=P \subset I$. This contradiction shows that $\bar{Q} \cap \bar{R}=\overline{0}$; i.e., $Q \cap R=I \cap R=P$.

We now proceed to prove Theorems 1 and 2 .

THEOREM 1. Let $R \subset S$ be a central integral extension of PI rings. Then $R \subset S$ has GD if and only if $R \subset S$ has SGD.

Proof. $(\Rightarrow)$ Suppose $R \subset S$ has GD, and let $t$ be any element of $S$. Let $P \subset P_{1}$ be two primes of $R$ and $Q_{1}$ a prime of $R[t]$ such that $Q_{1} \cap R=P_{1}$. Since $R[t] \subset S$ is a central integral extension, there is a prime $Q_{1}^{\prime}$ of $S$ with $Q_{1}^{\prime} \cap R[t]=Q_{1}$ by Lemma 2 . Note that $Q_{1}^{\prime} \cap R=P$; so there is a prime $Q^{\prime}$ of $S$ satisfying $Q^{\prime} \subset Q_{1}^{\prime}$ and $Q^{\prime} \cap R=P$ by GD in $R \subset S$. Let $Q=Q^{\prime}$ $\cap R[t]$. Again, $R[t] \subset S$ being a central extension gives that $Q$ is a prime ideal of $R[t]$. Furthermore, $Q \subset Q_{1}$ and $Q \cap R=P$. Thus $R \subset R[t]$ has GD for every $t \in S$.

$(\Leftarrow)$ Assume that $R \subset S$ has SGD. Suppose there are two primes $P \subset P_{1}$ of $R$ and a prime $Q_{1}^{\prime}$ of $S$ so that $Q_{1}^{\prime} \cap R=P_{1}$. Define $I$ as in Lemma 4 using $W=\left\{Q_{\alpha} \mid Q_{\alpha}\right.$ is a prime ideal of $\left.S, Q_{\alpha} \cap R=P\right\}$. Note that $R \subset S$ has LO 
by Lemma 2 ; hence, $W \neq \varnothing$. By Lemma 4 we will be done if we can show that $I \subseteq Q_{1}^{\prime}$. If this is not true, pick $t \in I-Q_{1}^{\prime}$ and consider $R[t]$. Let $Q_{1}=Q_{1}^{\prime} \cap R[t]$ which is prime in $R[t]$ as above. By GD in $R[t]$ there is a prime ideal $Q$ of $R[t]$ such that $Q \subset Q_{1}$ and $Q \cap R=P$. As before $R[t] \subset S$ has LO; so there exists a prime $Q^{\prime}$ of $S$ with $Q^{\prime} \cap R[t]=Q$. But then $Q^{\prime} \cap R=P$ gives $I \subset Q^{\prime}$ which says $t \in Q^{\prime} \cap R[t]=Q \subset Q_{1}=Q_{1}^{\prime}$ $\cap R[t]$. This contradicts our choice of $t$. Therefore, $I \subset Q_{1}^{\prime}$ as required.

THeOREM 2. Let $R$ be a PI ring integral over a central subring $C$. Then $C \subset R$ has GD if and only if $C \subset R$ has SGD.

Proof. $(\Rightarrow)$ Suppose $C \subset R$ has GD, and let $t$ be any element of $R$. Set $B=C[t]$. Let $P \subset P_{1}$ be two primes of $C$ such that there is a prime $Q_{1}$ of $B$ lying over $P_{1}$ (i.e., $P_{1}=Q_{1} \cap C$ ). Take $q_{1}$ to be an ideal of $R$ maximal with respect to $q_{1} \cap B \subseteq Q_{1}$. It is easy to see that $q_{1}$ is a prime ideal of $R$. It is also true that $q_{1} \cap C=P_{1}$. For $q_{1} \cap C \subseteq\left(q_{1} \cap B\right) \cap C \subseteq Q_{1} \cap C=P_{1}$. If $q_{1} \cap C \varsubsetneqq P_{1}$, there is a $z \in P_{1}-q_{1} \cap C$. Consider $\bar{R}=R / q_{1}$. Applying Lemma 1 to the rings $\bar{B} \subset \bar{R}$, we find that $\overline{z R} \cap \bar{B} \subseteq \bar{Q}_{1}$ which translates to $\left(z R+q_{1}\right) \cap\left(B+q_{1}\right) \subseteq Q_{1}+q_{1}$ in $R$. Taking the intersection of both sides with $B$, we get $\left(z R+q_{1}\right) \cap B \subseteq\left(Q_{1}+q_{1}\right) \cap B=Q_{1}$. But the ideal $\left(z, q_{1}\right)$ is properly larger than $q_{1}$, contradicting the maximality of $q_{1}$. Thus $q_{1} \cap C=P_{1}$.

By hypothesis there is a prime ideal $q$ of $R$ such that $q \subset q_{1}$ and $q \cap C=P$. Let $S=C-P$ and $T=B-Q_{1} . P$ and $Q_{1}$ are prime ideals of $C$ and $B$, respectively. Hence, $S T=\{s t \mid s \in S, t \in T\}$ is a multiplicatively closed subset of $B$. Note that $S T \cap(q \cap B)=\varnothing$ because elements of $S$ are regular $\bmod q$ in $R$, hence in $B$, and $q \subset q_{1}$ gives $q \cap B \subset q_{1} \cap B \subseteq Q_{1}$ so that $(q \cap B) \cap T=\varnothing$. Let $Q$ be an ideal of $B$ containing $q \cap B$ and maximal with respect to $Q \cap S T=\varnothing$. We note three things about $Q$ :

(1) $Q$ is a prime ideal of $B$.

(2) $Q \cap C=P$. For $Q \supseteq q \cap B$ implies $Q \cap C \supseteq(q \cap B) \cap C=P$. If $Q \cap C \supsetneqq P$, take any $s \in(Q \cap C)-P$ and any $t \in T$. Then $s \in S$ and $s t \in Q \cap S T$. Contradiction.

(3) $Q \subset Q_{1}$. If not, take $t \in Q-Q_{1}$ and any $s \in S$. Then $t \in T$ and $s t \in Q \cap S T$. Contradiction.

Therefore $C \subseteq B=C[t]$ has GD.

$(\Leftrightarrow)$ Suppose $C \subset R$ has SGD. Let $P \subset P_{1}$ be two primes of $C$ and $q_{1}$ a prime of $R$ lying over $P_{1}$ in $C$. Let $W=\left\{q_{\alpha} \mid q_{\alpha}\right.$ is prime in $R$ and $q_{\alpha} \cap C$ $=P\} . W \neq \varnothing$ since $C \subset R$ has LO by Lemma 2. Set $I=\cap\left\{q_{\alpha} \mid q_{\alpha} \in W\right\}$. By Lemma 4 it will be enough to show that $I \subset q_{1}$. If not, choose $t \in I$ so that $t$ is regular $\bmod q_{1}\left[2\right.$, p. 48]. Let $B=C[t]$, and let $Q_{1}$ be a prime ideal of $B$ with $Q_{1} \supseteq q_{1} \cap B$ and $Q_{1} \cap C=P_{1}$. (Just enlarge $q_{1} \cap B$, if necessary, to an ideal of $B$ maximal with respect to lying over $P_{1}$.) Apply the SGD hypothesis to find a prime ideal $Q$ of $B$ so that $Q \subset Q_{1}$ and $Q \cap C=P$. If we now take $q$ to be an ideal of $R$ maximal with respect to $q \cap B \subseteq Q$, the same argument of the first part of this proof shows that $q$ is prime in $R$ and $q \cap C=P$. So 
$q=q_{\alpha} \in W$; whence $I \subset q$, and $t \in q$. Thus $t \in q \cap B \subseteq Q \subset Q_{1}$ implies $t \in Q_{1}$. Now $Q_{1}$ is a prime minimal over the ideal $q_{1} \cap B$ in $B$. Otherwise, there is a prime $Q_{0}$ of $B$ such that $q_{1} \cap B \subseteq Q_{0} \subset Q_{1}$. This would give $P_{1}=\left(q_{1} \cap B\right) \cap C \subseteq Q_{0} \cap C \subseteq Q_{1} \cap C=P_{1}$, contradicting INC in $C$ $\subseteq B$. But $Q_{1}$ minimal over $q_{1} \cap B$ implies that $Q_{1}$ consists of elements which are zero-divisors $\bmod q_{1} \cap B$ by Lemma 3. So there exists $x \in B-q_{1} \cap B$ so that $x t \in q_{1} \cap B$. This contradicts the fact that $t$ is regular $\bmod q_{1}$ in $R$. Therefore $I \subset q_{1}$, and $C \subset R$ has GD.

COROllaRY (SCHELTER). If $R$ is a prime PI ring integral over an integrallyclosed central subring $A$, then $A \subseteq R$ has GD.

Proof. By Theorem 2, $A \subseteq R$ has GD if and only if $A \subseteq R$ has SGD. If $t \in R$, then $A[t]$ is a commutative subring of $R$ with no zero-divisors in $A$. The commutative going down theorem (see e.g. [6]) may be applied to see that $A \subseteq A[t]$ has GD.

\section{REFERENCES}

1. Stephen McAdam, Private communication.

2. Claudio Procesi, Rings with polynomial identities, Dekker, New York, 1973. MR 51 \#3214.

3. William Schelter, Integral extensions of rings satisfying a polynomial identity, J. Algebra 40 (1976), 245-257.

4. - Non-commutative affine P.I. rings are Catenary (to appear).

5. L. Rowen, Some results on the center of a ring with polynomial identity, Bull. Amer. Math. Soc. 79 (1973), 219-223. MR 46 \#9099.

6. I. Cohen and A. Seidenberg, Prime ideals and integral dependence, Bull. Amer. Math. Soc. 52 (1946), 252-261. MR 7, 410.

Department of Mathematics, University of Southern California, Los Angeles, CaliFORNIA 90007 